\title{
SÉRIES CLIMÁTICAS EM GRADE DE PRECIPITAÇÃO E TEMPERATURA DO AR EM REGIÃO DE RELEVO COMPLEXO
}

\author{
SANTOS, Janaina Cassiano dos - jana.cassiano@gmail.com \\ Universidade Federal Rural do Rio de Janeiro / UFRRJ
}

PRADO, Dayanne de Oliveira - dayanne_pradovr@hotmail.com Universidade Federal Rural do Rio de Janeiro / UFRRJ

LYRA, Gustavo Bastos - gblyra@gmail.com

Universidade Federal Rural do Rio de Janeiro / UFRRJ

SANTOS, Ednaldo Oliveira dos - edmeteoro@hotmail.com

Universidade Federal Rural do Rio de Janeiro / UFRRJ

\begin{abstract}
RESUMO: Avaliaram-se séries climáticas (1960-91) de precipitação e temperatura do ar mensal de produtos em grade em relação às séries desses elementos observadas em estações meteorológicas. As séries climáticas observadas foram obtidas nas estações do Instituto Nacional de Meteorologia, localizadas no estado do Rio de Janeiro, enquanto que as séries dos produtos em grade foram extraídas nos pontos de grade (resolução $0,5^{\circ} \mathrm{X}$ $\left.0,5^{\circ}\right)$ dos produtos do Global Precipitation Climatology Center (GPCC), The Global Historical Climatology Network (GHCN) ou Universidade de Delaware (UDEL) mais próximos das estações em estudo. Avaliou-se a precisão (coeficiente de determinação $r^{2}$ ) e exatidão (índice de concordância de Willmott - d e Raiz do Quadrado Médio do Erro - RQME) de cada produto em grade em relação às séries observadas. Os produtos em grade de precipitação (GPCC e UDEL) não tiveram precisão satisfatória $\left(r^{2}<0,54-\right.$ GPCC e $r^{2}<0,61$ - UDEL), contudo sua exatidão ( $d>0,59$ - GPCC e $d>0,58$ - UDEL) foi superior à precisão. Os erros observados para precipitação foram entre 59,5 e 125,8 $\mathrm{mm}$. As séries em grade de temperatura tiveram maior precisão $\left(r^{2}>0,41-\mathrm{GHCN}\right.$ e $r^{2}$ $>0,35$ - UDEL) e exatidão similar ( $d>0,58$ - GHCN e $d>0,65$ - UDEL) aos produtos de precipitação, com RQME entre 1,11 e 3,98 ${ }^{\circ} \mathrm{C}$. Foram identificadas associações que elevam o erro dos produtos em grade para a região, tais como, o elevado gradiente altitudinal da área de estudo e o efeito continentalidade/maritimidade. Dentre os produtos em grade de precipitação, o GPCC apresentou melhor desempenho (maior precisão e exatidão) em relação à UDEL na maior parte das estações, enquanto para temperatura do ar as séries da UDEL se sobressaíram em comparação ao GHCN. Portanto, faz-se necessário desenvolver produtos climáticos de precipitação e temperatura do ar em grade precisos e exatos com alta resolução para o estado do Rio de Janeiro.
\end{abstract}

PALAVRAS-CHAVE: Climatologia, Geoprocessamento, Gradeamento, Downscaling

CLIMATE SERIES IN PRECIPITATION AND AIR TEMPERATURE GRID IN COMPLEX RELIEF REGION

ABSTRACT: Monthly climatic time series (1960-1991) for precipitation and air temperature of gridded products were evaluated regarding to series observed in meteorological stations of Rio de Janeiro state. The observed climatic series were obtained from Brazilian National Institute of Meteorology. The gridded product series were extracted at grid points (resolution $0.5^{\circ} \times 0.5^{\circ}$ ) from Global Precipitation Climatology Center (GPCC), The Global Historical Climatology Network (GHCN) or University of Delaware (UDEL) near of stations under study. Precision (determination coefficient $-r^{2}$ ) and accuracy (Willmott agreement index - $d$ and Root Mean Square Error - RMSE) of each grid product were evaluated in relation to the observed series. The monthly gridded precipitation products (GPCC and UDEL) did not have satisfactory 
precision ( $r^{2}<0.54$ - GPCC and $r^{2}<0.61$ - UDEL), however its accuracy ( $d>0.59$ GPCC and $d>0.58$ - UDEL) was higher than precision. Observed precipitation errors were between 59.5 and $125.8 \mathrm{~mm}$. The gridded temperature data had higher precision $\left(r^{2}>0.41-\right.$ GHCN and $r^{2}>0.35-$ UDEL) and similar accuracy (d>0.58 - GHCN and $>$ 0.65 - UDEL) of the precipitation products, with RMSE between 1.11 and $3.98{ }^{\circ} \mathrm{C}$. Associations have been identified that increase the gridded product errors for region, such as elevated altitudinal gradient of study area and Maritime/Continental effect. Among gridded precipitation products, the GPCC showed better performance (greater precision and accuracy) than UDEL in most weather stations, while for air temperature it was observed that UDEL gridded series stood out in comparison to GHCN. Thus, it is necessary to develop precise and accurate gridded climate products with high resolution of precipitation and air temperature for Rio de Janeiro state.

KEYWORDS: Climatology, Geoprocessing, Railing, Downscaling

\section{INTRODUÇÃO}

Estudos climáticos se baseiam em séries de longo tempo de observações realizadas em estações meteorológicas/climatológicas de superfície, sendo elas automáticas ou convencionais. Esses estudos são de extrema importância para diversos setores, como por exemplo, agrícola, florestal, engenharias, geociências, ambiental, entre outros, pois permitem caracterizar tendências de curto (variabilidade sazonal e intraanual) e longo (variabilidade climática e mudanças climáticas) prazo, comparar essas tendências entre diferentes regiões, e assim descrever os padrões climáticos espaço-temporal de um território (ANDRIUCCI e NETO, 2006).

Apesar da sua importância, observações de longo tempo no Brasil restringem-se a séries não contínuas, de baixa qualidade, sem homogeneidade espaço-temporal e, ou com distribuição irregular no espaço (OLIVERIA-JÚNIOR et al., 2012; PRECINOTO et al., 2013). Associado a esses problemas, a baixa densidade de estações em diversas regiões do Brasil, limitam estudos climáticos regionais (SANTOS et al., 2016; TOSTES et al., 2017). Uma alternativa, principalmente para estudos climáticos em escala regional, são dados em grade, obtidos por interpolação em escala global de séries de precipitação e temperatura do ar observadas em estações meteorológicas de superfície (PETERSON et al., 1997; WILLMOTT et al., 2001; JONES e MOBERG, 2003; SCHNEIDER, 2013) e, ou simulados por modelos numéricos atmosféricos (KALNAY et al., 1996; KANAMITSU et al., 2002) ou mesmo derivados de sensores remotos orbitais (e.g. TRMM) (KAWANISHI et al., 1993; KAWANISHI et al., 2000).

Os produtos em grade de precipitação e temperatura do ar elaborados com dados do The Global Historical Climatology Network - GHCN (JONES e MOBERG, 2003), Global Precipitation Climatology Center - GPCC (SCHNEIDER et al., 2013) e os elaborados pela Universidade de Delaware (WILLMOTT et al., 2001), disponibilizados pela National oceanic and Atmospheric Administration (NOAA, 2016), no formato NetCDF, destacam-se devido à sua resolução e grade regular $\left(0,5^{\circ} \times 0,5^{\circ}\right)$, tamanho das séries ( $>50$ anos) e por não apresentarem falhas (TOSTES et al., 2017).

Os dados em grade podem ser obtidos por interpolação espacial de séries de observações de redes de estações meteorológicas de superfície localizadas no globo terrestre. A capacidade dos métodos de interpolação espacial representarem os padrões de distribuição de determinado fenômeno ou elemento é influenciado pela densidade das amostras a serem interpoladas, sua 
homogeneidade espacial, se ocorre anisotropia, sua variabilidade espacial, características do relevo, entre outros (LI e HEAP, 2008; ALVES e VECCHIA, 2011; LY et al., 2013; KELLER et al., 2015). Em regiões com relevo complexo, a elevada variabilidade dos elementos climáticos pode induzir erros significativos no processo de interpolação espacial (NINYEROLA et al., 2006; Di PIAZZA et al., 2011). Assim, é essencial a avaliação da precisão e exatidão das interpolações em relação as observações das estações de superfície terrestre.

O objetivo do trabalho foi avaliar a precisão e exatidão de séries climáticas mensais em grade de precipitação e de temperatura do ar para o período de 1961-1990 (normal climatológica) em condições de relevo complexo do estado do Rio de Janeiro.

\section{MATERIAL E MÉTODOS}

A área de estudo compreendeu o estado do Rio de Janeiro, localizado na região Sudeste do Brasil, entre as latitudes $20^{\circ} 44^{\prime}$ e $23^{\circ} 22^{\prime} \mathrm{S}$ e as longitudes $40^{\circ} 57^{\prime}$ e $44^{\circ}$ 53' W e com altitudes entre 0 e 2787 m a.n.m (Figura 1). O Estado apresenta relevo complexo, caracterizado por predominância de planície litorânea e planalto, além de algumas serras, entre elas a serra dos órgãos, serra do Mar, serra das Araras, entre outras (BRITO et al., 2016). O Rio de Janeiro faz divisa com São Paulo (Oeste), Minas Gerais (Norte) e Espírito Santo (Nordeste), sendo costeado pelo oceano Atlântico a Leste e Sul. O Estado, com base em aspectos físicos e sociais, foi subdividido em oito regiões administrativas de governo: Baixadas Litorâneas, Centro-Sul Fluminense, Costa Verde, Médio Paraíba, Metropolitana, Noroeste Fluminense, Norte Fluminense e Serrana (CEPERJ, 2009).

Utilizaram-se séries mensais observadas e em grade de precipitação e temperatura do ar no período de 1961 a 1990 (normal climatológica). As séries de precipitação e temperatura do ar observadas foram obtidas de onze estações meteorológicas convencionais, pertencentes ao Instituto Nacional de Meteorologia (INMET): ID1 - Angra dos Reis, ID 2 - Bangu, ID 3 - Campos dos Goytacazes, ID 4 - Carmo, ID 5 - Cordeiro, ID 6 - Itaperuna, ID 7 - Nova Friburgo, ID 8 - Resende, ID 9 - Rio de Janeiro, ID 10 - Santa Maria Madalena e 11 - Vassouras. 


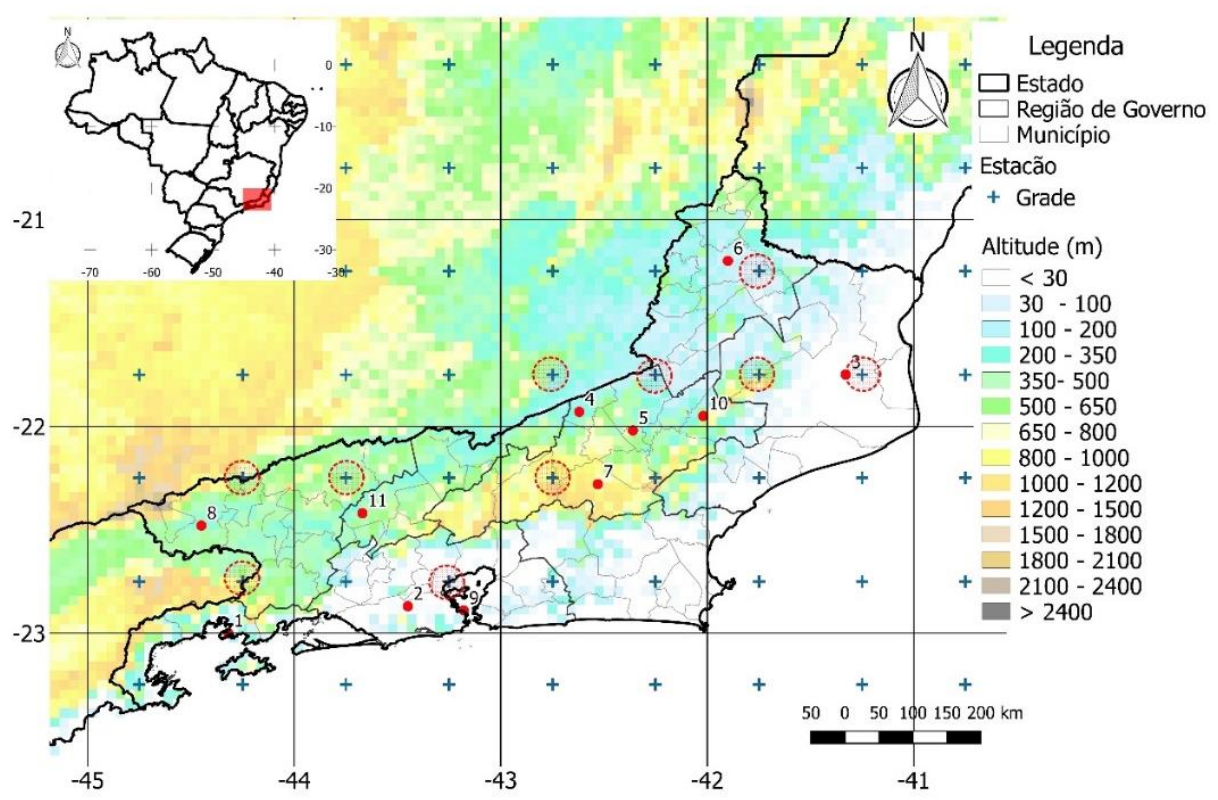

Figura 1 - Distribuição das estações meteorológicas e pontos de grade do Global Precipitation Climatology Center (GPCC), Global Historical Climate Network (GHCN) e Universidade de Delaware (UDEL) nas regiões de governo do estado do Rio de Janeiro e áreas limítrofes e altitude $(\mathrm{m})$. As séries dos pontos de grade destacados em círculos vermelhos foram usados para comparação com as séries das estações meteorológicas.

Devido parte das estações meteorológicas apresentar séries de temperatura do ar inferiores a 15 anos, foram utilizadas apenas oito estações meteorológicas (ID1, ID3, ID4, ID5, ID6, ID8, ID9, ID10) nas análises dos produtos de temperatura do ar.

As séries mensais de precipitação observadas foram submetidas à análise de qualidade dos dados. A análise de qualidade dos dados remete a obtenção de séries contínuas, homogêneas e sem falhas. A qualidade dos dados foi avaliada com base na análise exploratória $(A E)$ dos dados e no método de regressão linear simples (RLS) (OLIVEIRA-JÚNIOR et al., 2012; PRECINOTO et al., 2012). $\mathrm{Na} A E$ foram identificados os outliers, as observações fora do intervalo interquartis e os valores nulos de precipitação.

Após a $A E$, aplicou-se a análise da tendência espaço-temporal, que permitiu definir quais os valores espúrios de precipitação, que foram consequentemente retirados da série. A análise da tendência espaço-temporal foi realizada pelo método $\operatorname{RLS}\left(Y_{i}=\beta_{0}+\beta_{1} X_{i}\right)$, em que, $Y(\mathrm{~mm})$ é a série de precipitação mensal da estação em análise, $X(\mathrm{~mm})$ é a série de referência dessa estação, $\beta_{0}$ e $\beta_{1}$, são os coeficientes da regressão, respectivamente, intercepto e inclinação, e o termo subscrito i representa a i-ésima observação (OLIVEIRA-JÚNIOR et al., 2012). As observações identificadas na AE e fora do intervalo de confiança da RLS ( $p<0,05$ ), foram consideradas espúrias e retiradas da série.

Para obtenção da série de precipitação de referência $(X)$ de cada estação (Y) submetida à análise de qualidade foram primeiramente identificadas as estações com precipitações mensais homogêneas. Os grupos de estações com precipitação homogênea foram determinados pela análise de agrupamento - AA 
(cluster - método hierárquico aglomerativo de Ward) (WARD, 1963; LYRA et al., 2006; 2014).

Definidos os grupos de estações com precipitações homogêneas foi gerada uma matriz de correlação de precipitações mensais das estações de cada grupo. A partir da matriz de correlação foram escolhidas entre três e cinco outras estações de um mesmo grupo homogêneo e, que apresentaram o coeficiente de correlação de Pearson ( $r$ ) acima de 0,7 , preferencialmente aquelas que apresentaram este coeficiente o mais próximo possível de 1,0 (CORREIA et al., 2013). Os valores médios das observações das precipitações das estações selecionadas foram considerados representativos da tendência climática da região e constituíram a série de referência $(X)$.

As falhas de observações da série original ou decorrentes do controle de qualidade dos dados foram preenchidas também pelo método da RLS (KITE, 1988; OLIVEIRA-JÚNIOR et al., 2012; PRECINOTO et al., 2012).

Os dados em grade para o mesmo período dos dados observados foram obtidos por meio de interpolações espaciais de séries de observações mensais de redes de estações meteorológicas de superfície localizadas no globo terrestre. Os produtos em grade avaliados foram elaborados pela Universidade de Delaware (precipitação e temperatura do ar) versão 3.01, e precipitação obtida no Global Precipitation Climatology Center (GPCC) - Total Full V6 e temperatura do ar no Global Historical Climate Network (GHCN) - Versão 2. Esses produtos são disponibilizados pela National oceanic and Atmospheric Administration (NOAA) no formato NetCDF, com resolução espacial de $0,5^{\circ} \times 0,5^{\circ}$ (NOAA, 2016).

O GHCN é um banco de dados de temperatura do ar que integra resumos climáticos de estações de superfície, que são submetidas a um controle de qualidade. Os dados provêm de mais de 31 diferentes fontes com séries (redes de estações meteorológicas de superfície) entre 1948-2010. As séries do GHCN são disponíveis também como produtos em grade $\left(0,5^{\circ} \times 0,5^{\circ}\right)$, que abrangem todo o globo terrestre (LAWRIMORE et al., 2011). O GPCC fornece dados em grade $\left(0,5^{\circ} \times 0,5^{\circ}\right)$ da precipitação para todo o Planeta no período de 1948 2010, com finalidade de monitoramento e pesquisa de clima terrestre (SCHNEIDER et al., 2013). Os produtos em grade $\left(0,5^{\circ} \times 0,5^{\circ}\right)$ elaborados pela Universidade Delaware (UDEL) são obtidos pela interpolação espacial de séries climáticas de precipitação e temperatura do ar disponibilizadas em diversas fontes, algumas com mais de 100 anos de observações (MATSUURA e WILLMOTT, 2009).

Para as avaliações dos produtos em grade foram extraídas nos pontos de grade localizados mais próximos das 11 estações meteorológicas do INMET as séries mensais de precipitação (GPCC e UDEL) ou das oito estações com séries de temperatura do ar (GHCN e UDEL). A extração das séries na grade em formato NetCDF foi realizada com auxílio do programa MeteoInfo (WANG, 2014). Para avaliação das séries de precipitação e temperatura do ar mensal foi aplicada a análise de regressão linear $\left(Y=\beta_{0}+\beta_{1} X\right)$ entre as séries observadas $(X)$ e em grade $(Y)$. A partir da análise de regressão foi determinado o coeficiente de determinação $\left(r^{2}\right)$. Nesse caso, o $r^{2}$ é uma forma de avaliar a precisão dos dados em grade (interpolados) e varia de 0 a 1. Quanto mais próximo de 1 for o valor de $r^{2}$, mais preciso (menor a dispersão dos dados) é o dado em grade (WILLMOTT, 1981). 
Além da análise de regressão, foi determinada também a Raiz do Quadrado Médio do Erro (RQME) (Equação 1) e o índice de concordância de Willmott (d) (Equação 2).

$$
\begin{gathered}
R Q M E=\left[\frac{\sum_{i=1}^{n}\left(P_{i}-O_{i}\right)^{2}}{n}\right]^{1 / 2} \\
d=1-\left[\frac{\sum_{i=1}^{n}\left(P_{i}-O_{i}\right)^{2}}{\sum_{i=1}^{n}(|P i-O|+|O i-O|)^{2}}\right]
\end{gathered}
$$

O índice de concordância de Willmott representa a concordância (exatidão) das séries em grade em relação às séries observadas. $O$ índice d varia de 0 a 1 , sendo a maior exatidão à medida que os valores se aproximam de 1.

O desempenho dos dados interpolados em grade foi avaliado pelo índice de confiança (c) proposto por Camargo e Sentelhas (1997) (Equação 3). O índice c permite avaliar conjuntamente a precisão e a exatidão dos dados em grade, sendo obtido pelo produto do coeficiente de correlação de Pearson ( $r$ ) pelo índice de concordância (d).

$c=r * d$ 1997).

O índice c é caracterizado segundo a Tabela 1 (CAMARGO e SENTELHAS,

Tabela 1 - Classificação do índice de confiança (c) de Camarco e Setenlhas (1997).

\begin{tabular}{cc}
\hline Valor de "c" & Desempenho \\
\hline$>0,85$ & Ótimo \\
0,76 a 0,85 & Muito Bom \\
0,66 a 0,75 & Bom \\
0,61 a 0,65 & Mediano \\
0,51 a 0,60 & Sofrível \\
0,41 a 0,50 & Mau \\
$<0,41$ & Péssimo \\
\hline
\end{tabular}

\section{RESULTADOS E DISCUSSÃO}

\subsection{PRECIPITAÇÃO}

O coeficiente de determinação $\left(r^{2}\right)$ da regressão linear entre as séries de precipitação observadas e em grade da UDEL variou entre 0,15 e 0,54, respectivamente, para Rio de Janeiro e Itaperuna (Tabela 2). A precisão para o produto em grade do GPCC (Tabela 3) foi igual ou superior ao da UDEL em todas as estações avaliadas, exceto em Resende e Rio de Janeiro, onde o produto da UDEL foi mais preciso. Contudo, nessas estações as diferenças entre os $r^{2}$ dos dois produtos foram inferiores a 7,5\%. Os menores $r^{2}(<0,30)$ foram observados para as séries de precipitação em grade de Angra dos Reis e Rio de Janeiro, ambas localizadas próximas $(<5 \mathrm{~km}$ ) ao ambiente costeiro e com altitudes inferiores à $50 \mathrm{~m}$. As séries de precipitação de Itaperuna apresentaram as maiores precisões ( $r^{2}=0,54$ - UDEL e 0,61 - GPCC), seguida das séries de Nova Friburgo ( $r^{2}=0,50-$ UDEL e 0,54 - GPCC). 
A exatidão das séries em grade da UDEL, com base no d de Willmott, foi inferior ou igual na maior parte das estações em relação às séries do GPCC. Apenas na estação do Rio de Janeiro, o produto do GPCC foi mais exato. O índice d foi de 0,59 - UDEL e 0,58 - GPCC, para as séries do Rio de Janeiro, e 0,85 - UDEL e 0,88 - GPCC, para as séries de Itaperuna. As menores exatidões foram observadas para as séries em grade de Angra dos Reis e Rio de Janeiro, similar ao apresentado para precisão. De forma geral, a exatidão dos produtos de precipitação foi superior à sua precisão.

Tabela 2 - Análise estatística, com o coeficiente de determinação $\left(r^{2}\right)$, índice de concordância de Willmot (d), índice de desempenho (c) e Raiz do Quadrado Médio do Erro (RQME, $\mathrm{mm}$ ou \%) das séries em grade da Universidade de Delaware em relação às séries observadas de precipitação.

\begin{tabular}{|c|c|c|c|c|c|}
\hline NOME & $\mathbf{r}^{2}$ & $\mathbf{d}$ & C & $\begin{array}{l}\text { RQME } \\
(\mathrm{mm})\end{array}$ & $\begin{array}{c}\text { RQME } \\
(\%)\end{array}$ \\
\hline Angra dos Reis & 0,27 & 0,66 & 0,34 & 125,8 & 79,7 \\
\hline Bangu & 0,36 & 0,74 & 0,45 & 93,7 & 81,2 \\
\hline Campos dos Goytacazes & 0,42 & 0,78 & 0,50 & 67,7 & 83,6 \\
\hline Carmo & 0,35 & 0,74 & 0,44 & 93,2 & 82,6 \\
\hline Cordeiro & 0,43 & 0,79 & 0,51 & 80,8 & 75,7 \\
\hline Itaperuna & 0,54 & 0,85 & 0,63 & 66,3 & 69,0 \\
\hline Nova Friburgo & 0,50 & 0,83 & 0,59 & 77,0 & 76,0 \\
\hline Resende & 0,45 & 0,79 & 0,53 & 94,4 & 75,4 \\
\hline Rio de Janeiro & 0,15 & 0,59 & 0,23 & 100,8 & 120,2 \\
\hline Santa Maria Madalena & 0,45 & 0,80 & 0,53 & 82,1 & 69,4 \\
\hline Vassouras & 0,36 & 0,75 & 0,45 & 90,7 & 87,3 \\
\hline
\end{tabular}

Tabela 3 - Análise estatística, com o coeficiente de determinação $\left(r^{2}\right)$, índice de concordância de Willmot (d), índice de desempenho (c) e Raiz do Quadrado Médio do Erro (RQME, mm ou \%) das séries em grade do Global Precipitation Climatology Center (GPCC) em relação às séries observadas de precipitação.

\begin{tabular}{llllll}
\hline Nome & $\mathbf{r}^{\mathbf{2}}$ & $\mathbf{d}$ & $\mathbf{C}$ & $\begin{array}{l}\text { RQME } \\
(\mathbf{m m})\end{array}$ & $\begin{array}{l}\text { RQME } \\
(\mathbf{\%})\end{array}$ \\
\hline Angra dos Reis & 0,27 & 0,66 & 0,34 & 124,3 & 78,7 \\
Bangu & 0,36 & 0,75 & 0,45 & 94,3 & 81,8 \\
Campos dos Goytacazes & 0,48 & 0,81 & 0,56 & 60,4 & 74,5 \\
Carmo & 0,38 & 0,76 & 0,47 & 89,3 & 79,1 \\
Cordeiro & 0,52 & 0,82 & 0,59 & 74,7 & 70,0 \\
Itaperuna & 0,61 & 0,88 & 0,69 & 59,5 & 61,9 \\
Nova Friburgo & 0,54 & 0,84 & 0,62 & 72,8 & 71,8 \\
\hline Resende & 0,44 & 0,79 & 0,53 & 94,7 & 75,6 \\
Rio de Janeiro & 0,14 & 0,58 & 0,22 & 105,0 & 125,2 \\
\hline Santa Maria Madalena & 0,48 & 0,80 & 0,56 & 75,2 & 63,6 \\
Vassouras & 0,37 & 0,76 & 0,46 & 92,0 & 88,5 \\
\hline
\end{tabular}

Tostes et al. (2017) também observaram maior exatidão dos produtos em grade de precipitação do GPCC e UDEL para o período de 1970-2000 em 
relação a sua precisão para séries de precipitação de estações no Acre e sul da Amazônia. A precisão $\left(0,62 \leq r^{2} \leq 0,91\right.$ - GPCC e 0,52 $\leq r^{2} \leq 0,89$ - UDEL) e exatidão $\left(0,87 \leq d \leq 0,97\right.$ - GPCC e $0,82 \leq r^{2} \leq 0,97$ - UDEL) obtidas por esses autores para os produtos de precipitação foram superiores aos observados para a região de estudo do presente trabalho, com exceção das séries da UDEL nas estações de Itaperuna e Nova Friburgo. Zhu et al. (2015) avaliaram os produtos em grade de precipitação do GPCC e UDEL para uma região árida da China (Xinjiang) para o período de $1979-2010$ e reportaram $r^{2}$ inferiores ou superiores, dependendo da estação, aos observados no presente trabalho e entre 0,09 a 0,68 - UDEL e 0,08 a 0,72 - GPCC.

O RQME das séries de precipitação em grade da UDEL variou entre 66,3 (Itaperuna) e 125,8 mm (Angra dos Reis), que representaram, respectivamente, 69 e 79,7 \% da média da precipitação dessas estações (RQME, \%). Contudo, a estação do Rio de Janeiro, apesar de menor RQME absoluto que Angra dos Reis, apresentou maior RQME\% de todas as estações avaliadas (120,2 \%). Para o GPCC, o RQME foi menor que o obtido para as séries da UDEL nas estações de Angra dos Reis, Campos, Cordeiro, Itaperuna, Nova Friburgo e Santa Maria Madalena, enquanto que nas demais estações o RQME foi menor na UDEL. $O$ RQME para o GPCC foi de 59,5 (61,9 \%) a 124,3 mm (78,7 \%), respectivamente, para Itaperuna e Angra dos Reis. Para os dois produtos, os maiores RQME absoluto (> $120 \mathrm{~mm}$ ) ocorreram em Angra dos Reis, mas em termos relativos, os maiores erros ( $>120 \%$ ) foram observados no Rio de Janeiro.

Os erros observados por Tostes et al. (2017) para os produtos em grade de precipitação (GPCC e UDEL) também foram inferiores aos obtidos no presente trabalho (35,6 - 79,6 mm - GPCC e 35,4 - 79,3 mm - UDEL), que representaram entre 22,7 e $50,5 \%$ da média das precipitações dessas localidades. Zhu et al. (2015) encontraram RQME da UDEL entre 25,9 e 163,8 $\mathrm{mm}$ e para o GPCC entre 17,3 e 161,4 mm.

A precipitação avaliada com base no índice c de Camargo e Sentelhas (precisão e exatidão) indicou que o desempenho das séries se alterou pouco, análogo ao padrão observado para a exatidão, com as séries do GPCC (exceto Rio de Janeiro) com desempenho maior ou igual (Angra dos Reis, Resende e Santa Maria Madalena) as séries da UDEL. Para as séries em grade da UDEL, 0 desempenho (índice c) variou entre 0,23 (Rio de Janeiro) e 0,63 (Itaperuna), enquanto para as séries do GPCC, o índice c variou entre 0,22 (Rio de Janeiro) e 0,69 (Itaperuna). Em ambos os produtos, Rio de Janeiro e Angra dos Reis tiveram séries com menor desempenho (c <0,34), que segundo a classificação desse índice, indicou desempenho Péssimo. Os melhores desempenhos, $\mathrm{c}=$ 0,63 (Mediano) - UDEL e 0,69 (Bom) - GPCC, foram observados para as séries de Itaperuna.

O desempenho do GPCC e UDEL no estado do Acre e sul do Amazonas para o estudo de Tostes et al. (2017) foi superior ao do presente trabalho e classificados entre Bom $(0,68)$ e Ótimo $(0,93)$ para as séries do GPCC, sendo inferior para a UDEL e entre Sofrível $(0,59)$ e Ótimo $(0,91)$. Zhu et al. $(2015)$ obtiveram maior precisão $\left(r^{2}\right)$ na maior parte das estações para o produto da UDEL, enquanto os menores RQME foram observados para o produto do GPCC.

Os melhores resultados obtidos por Tostes et al. (2017) para os produtos do GPCC e UDEL estão relacionados ao menor gradiente altitudinal do Acre e sul 
do Amazonas em relação ao estado do Rio de Janeiro. No estado do RJ, além de maior gradiente altitudinal, que induz a efeitos orográficos, as precipitações são também influenciadas pelo efeito continentalidade/maritimidade. Esses fatores induzem a maior variabilidade espacial das precipitações no estado do RJ (BRITO et al., 2016) em relação ao observado no Acre e sul do Amazonas. A maior variabilidade espacial das precipitações no RJ não consegue ser representada adequadamente em uma grade de resolução $0,5^{\circ}$, que é o caso dos produtos do GPCC e UDEL. Zhu et al. (2015) comentam que o erro do GPCC e UDEL aumentam em regiões montanhosas, principalmente, para altitudes maiores que $1.500 \mathrm{~m}$.

Para os produtos de precipitação foram observados dois padrões: i) subestimativa (Figura 2c e 2d), que ococorreu na maior parte das estações, principalmente, para o GPCC e ii) superestimava/subestimativa (Figura 2a e 2b). A tendência de superestimava (subestimativa) para valores de precipitação maiores (menores) que aproximadamente 50 a $100 \mathrm{~mm}$, ocorreu na estação do Rio de Janeiro, para as séries do GPPC e da UDEL, e Campos dos Goytacazes e Itaperuna apenas para UDEL. Zhu et al. (2015) também relatam padrão de subestimativa dos produtos em grade do GPCC e UDEL.
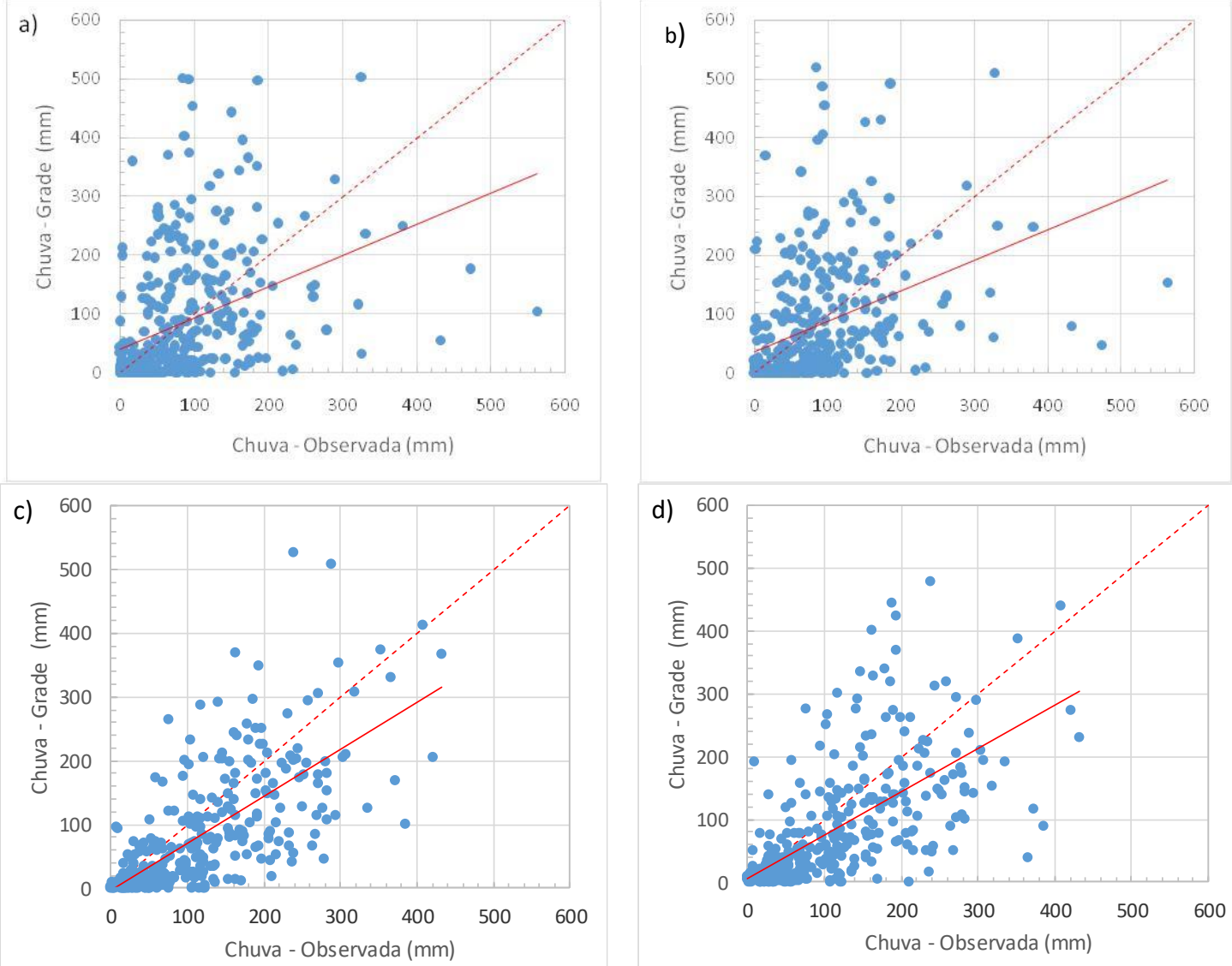

Figura 2 - Relação entre as séries observadas e em grade de precipitação para as estações Rio de Janeiro (a, b) e Cordeiro (c, d) dos produtos em grade da Universidade de Delaware (b, d) e do Global Precipitation Climatology Center (a, c). 
A tendência foi de aumento do erro a medida que os valores de chuva aumentaram (Figura 2), isso indicou que no verão os erros dos produtos em grade foram maiores. No verão, a interação do sistema de brisa marítima e o relevo contribuem com as chuvas (BRITO et al., 2016), visto que ocorre aporte de umidade da brisa em direção as encostas das serras voltadas para o oceano Atlântico, o que potencializa o efeito orográfico. Os maiores erros foram observados nas estações que apresentaram os maiores desníveis relativos (diferença entre a altitude da estação e do ponto de grade) entre a localidade da estação e o ponto de grade considerado e as maiores distâncias entre a estação e o ponto de grade, para ambos os produtos GPCC e UDEL (Figura 3a e b).
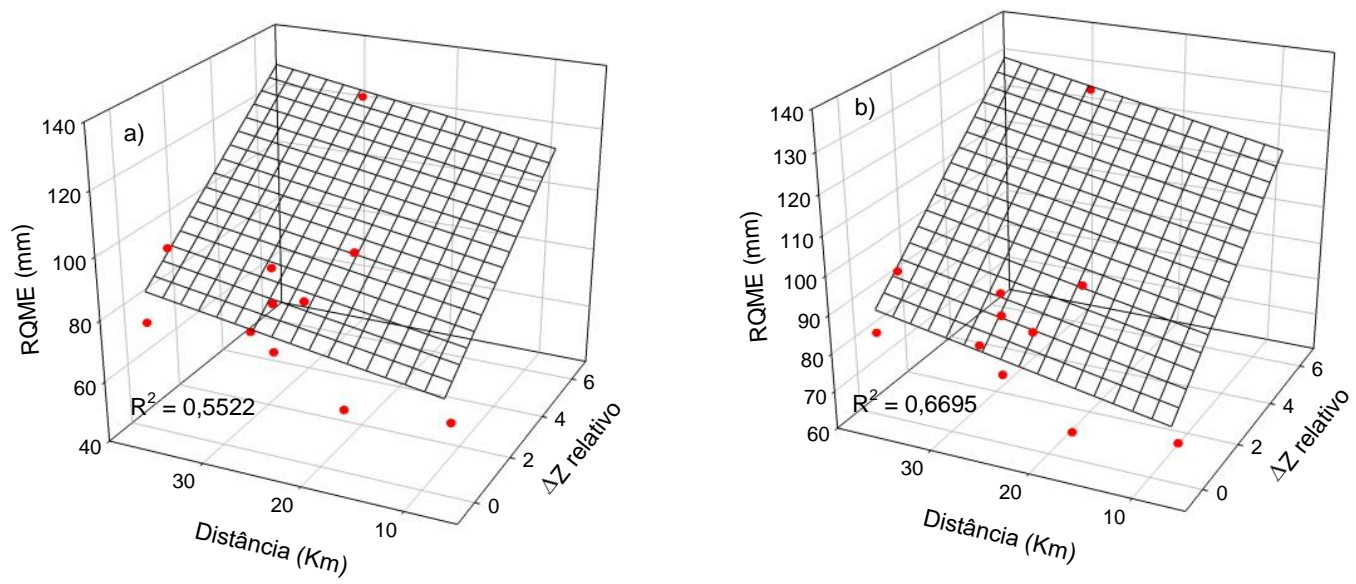

Figura 3 - Relação entre a Raiz do Quadrado Médio do Erro (RQME, mm), a distância (d, $\mathrm{km})$ e a diferença relativa entre a altitude $(\Delta Z)$ da estação e a do ponto de grade do a) GPCC e b) Universidade de Delaware - UDEL.

\subsection{TEMPERATURA DO AR}

O coeficiente de determinação $\left(r^{2}\right)$ da regressão linear simples entre os valores de temperatura do ar observados e em grade para o produto da UDEL variou entre 0,41 (Angra dos Reis) e 0,84 (Carmo e Itaperuna) (Tabela 4). A precisão, indicada pelo $r^{2}$, para o produto em grade da UDEL foi superior ao do GHCN (Tabela 5) em todas as estações avaliadas. Os menores $r^{2}(\leq 0,43)$, para ambos os produtos em grade, foram observados para as séries de Angra dos Reis e Rio de Janeiro, as duas estações próximas ao ambiente costeiro e com baixas altitudes. As maiores precisões $\left(r^{2} \geq 0,76\right)$ foram observadas para as séries de temperatura do ar de Campos dos Goytacazes, Cordeiro e Itaperuna, para os dois produtos avaliados e, no caso da UDEL, além dessas, Carmo e Santa Maria Madalena também apresentaram $r^{2}$ entre os maiores.

Tostes et al. (2017) avaliaram os produtos em grade de temperatura do ar do GHCN e UDEL em relação as séries observadas nas estações localizadas no estado do Acre (Rio Branco, Tarauacá e Cruzeiro do Sul) e no Amazonas (Lábrea e Eirunepé) para o período de 1971-2010. Os autores observaram precisão ( $\left.r^{2}\right)$ dos dois produtos inferiores ou próximos aos obtidos no presente trabalho e entre 0,19 e 0,61 para o GHCN e de 0,03 a 0,79 para a UDEL. Na maior parte das estações (três), o produto da UDEL mostrou maior precisão em relação ao GHCN, resultado análogo ao do presente trabalho. 
Tabela 4 - Análise estatística com coeficiente de determinação $\left(r^{2}\right)$, índice de concordância de Willmott (d), índice de desempenho (c) e Raiz do Quadrado Médio do Erro (RQME, ${ }^{\circ} \mathrm{C}$ ou \%) das séries em grade da Universidade de Delaware - UDEL em relação às séries observadas de temperatura do ar.

\begin{tabular}{lccccc}
\hline NOME & $\mathbf{r}^{\mathbf{2}}$ & $\mathbf{d}$ & $\mathbf{C}$ & $\begin{array}{c}\text { RQME } \\
\left({ }^{\circ} \mathbf{C}\right)\end{array}$ & $\begin{array}{c}\text { RQME } \\
(\mathbf{\%})\end{array}$ \\
\hline Angra dos Reis & 0,41 & 0,66 & 0,42 & 2,89 & 12,3 \\
Campos dos Goytacazes & 0,81 & 0,80 & 0,72 & 2,15 & 8,7 \\
Carmo & 0,84 & 0,65 & 0,59 & 3,98 & 16,9 \\
Cordeiro & 0,79 & 0,86 & 0,76 & 1,74 & 8,0 \\
Itaperuna & 0,84 & 0,94 & 0,86 & 1,11 & 4,6 \\
Resende & 0,63 & 0,86 & 0,68 & 1,67 & 7,4 \\
Rio de Janeiro & 0,43 & 0,66 & 0,43 & 2,65 & 11,0 \\
Santa Maria Madalena & 0,81 & 0,90 & 0,81 & 1,43 & 6,8 \\
\hline
\end{tabular}

Tabela 5 - Análise estatística, com coeficiente de determinação $\left(r^{2}\right)$, índice de concordância de Willmott (d), índice de desempenho (c) e Raiz do Quadrado Médio do Erro (RQME, ${ }^{\circ} \mathrm{C}$ ou \%) das séries em grade do Global Historical Climate Network (GHCN) em relação às séries observadas de temperatura do ar.

\begin{tabular}{lccccc}
\hline Nome & $\mathbf{r}^{\mathbf{2}}$ & $\mathbf{d}$ & $\mathbf{C}$ & $\begin{array}{c}\text { RQME } \\
\left({ }^{\circ} \mathbf{C}\right)\end{array}$ & $\begin{array}{c}\text { RQME } \\
(\mathbf{\%})\end{array}$ \\
\hline Angra dos Reis & 0,36 & 0,71 & 0,43 & 2,30 & 9,8 \\
Campos dos Goytacazes & 0,77 & 0,80 & 0,70 & 2,07 & 8,4 \\
Carmo & 0,67 & 0,77 & 0,63 & 2,49 & 10,6 \\
Cordeiro & 0,76 & 0,89 & 0,77 & 1,50 & 7,0 \\
Itaperuna & 0,77 & 0,83 & 0,73 & 1,88 & 7,8 \\
Resende & 0,46 & 0,81 & 0,55 & 1,88 & 8,4 \\
Rio de Janeiro & 0,35 & 0,58 & 0,34 & 3,29 & 13,7 \\
Santa Maria Madalena & 0,67 & 0,84 & 0,69 & 1,77 & 8,5
\end{tabular}

Similar ao padrão observado para precisão, a exatidão das séries em grade da UDEL, indicada pelo índice de concordância de Willmott (d), foi superior ou igual (Campos dos Goytacazes) na maior parte das estações ao produto do GHCN. As exceções ocorreram nas estações de Angra dos Reis, Carmo e Cordeiro, onde o produto do GHCN foi mais exato. O índice d para as séries da UDEL variou entre 0,65 (Carmo) e 0,94 (Itaperuna) e para o GHCN de 0,58 (Rio de Janeiro) a 0,89 (Cordeiro). As menores exatidões ( $d<0,71$ ) foram proporcionadas pelas séries de grade representativas de Angra dos Reis e Rio de Janeiro, assim como o observado para precisão. Para UDEL, Carmo também mostrou exatidão entre as menores. De forma geral, a exatidão dos produtos avaliados foi superior à sua precisão, com exceção de Carmo e Campos dos Goytacazes, para a série da UDEL, onde a precisão foi maior que exatidão.

A exatidão desses produtos reportado por Tostes et al. (2017) para estações no Acre e Amazonas variaram de 0,52 $\leq \mathrm{d} \leq 0,88$ para o $\mathrm{GHCN}$ e entre $0,25 \leq \mathrm{d} \leq 0,79$ para a UDEL. A UDEL também mostrou maior exatidão na maior parte (três estações) das estações avaliadas desses Estados.

O erro dos produtos em grade (RQME) da UDEL para temperatura do ar variou entre 1,11 (Itaperuna) e 3,98 ${ }^{\circ} \mathrm{C}$ (Carmo), que representaram, 
respectivamente, 4,6 e 16,9 \% da média da temperatura do ar dessas estações (RQME, \%). Para o GHCN, o RQME foi menor que o observado para as séries da UDEL nas estações de Angra dos Reis, Campos dos Goytacazes, Carmo e Cordeiro, nas demais estações, o RQME foi maior para as séries do GHCN. O RQME para o GHCN foi de 1,50 (7 \%) a 3,29 ${ }^{\circ} \mathrm{C}(13,7 \%)$, respectivamente, para Cordeiro e Rio de Janeiro. Para os dois produtos, os maiores RQME $(>2,65$ $\left.{ }^{\circ} \mathrm{C}\right){ }^{\circ}$ Correram em Angra dos Reis, Rio de Janeiro e Carmo, enquanto os menores $\left(<1,74^{\circ} \mathrm{C}\right)$ foram observados em Cordeiro, Itaperuna e Santa Maria Madalena.

Erros inferiores ao do presente trabalho foram observados por Tostes et al. (2017) e entre 0,8 (3\%) e 1,6 ${ }^{\circ} \mathrm{C}(6 \%)$ - GHCN e 1,0 (3,9 \%) e 1,6 ${ }^{\circ} \mathrm{C}(6$ $\%)$ - UDEL. No estado do Acre e Sul do Amazonas observa-se baixa variabilidade espacial da temperatura do ar, devido à proximidade com o Equador e o baixo gradiente de altitude (50 - $609 \mathrm{~m}$ ), que também induzem a baixa amplitude da temperatura ao longo do ano (TOSTES et al., 2017). No estado do Rio de Janeiro, a proximidade com o Trópico de Capricórnio e o elevado gradiente de altitude $(0$ até $2791 \mathrm{~m})$, associadas ao efeito continentalidade/maritimidade, induzem a maior variabilidade espacial e temporal das temperaturas do ar em relação ao Acre e o Amazonas. Essa elevada variabilidade da temperatura no estado do Rio de Janeiro ocorre em distâncias inferiores a resolução da grade do $\operatorname{GHCN}$ e UDEL $\left(0,5^{\circ} \sim 54 \mathrm{~km}\right)$, o que resulta em maiores erros médios desses produtos para a região de estudo em relação ao Acre e Amazonas.

Ao avaliar o desempenho (precisão e exatidão), com base no índice c de Camargo e Sentelhas, o padrão observado para a exatidão se repete na maior parte das séries em grade, com desempenho superior para UDEL (entre 0,42 Angra do Reis e 0,86 - Itaperuna) em comparação com as séries do GHCN (entre 0,34 - Rio de Janeiro e 0,77 - Cordeiro), exceto para Angra dos Reis, Carmo e Cordeiro, onde o GHCN apresentou melhor desempenho. Contudo, 0 melhor desempenho do GHCN nessas estações mostrou diferenças entre o índice c dos dois produtos inferiores a 6,5 \%. Rio de Janeiro e Angra dos Reis tiveram séries com menor desempenho (c $<0,43)$, que segundo a classificação desse índice, indicou desempenho entre Péssimo (Rio de Janeiro - GHCN) e Mau (Rio de Janeiro - UDEL e Angra dos Reis - GHCN e UDEL). Os melhores desempenhos $(0,76 \leq c \leq 0,85$ - Bom e $c>0,85$ - Muito Bom) foram observados para as séries de Cordeiro, Itaperuna e Santa Maria Madalena para as séries da UDEL e apenas Cordeiro para o produto do GHCN.

O desempenho dos produtos do GHCN para o Acre e Amazonas avaliados por Tostes et al. (2017) variaram de 0,27 a 0,69, com a maior parte das estações caracterizadas como, respectivamente, Péssimo e Bom, enquanto para o produto da UDEL essa variação foi de 0,04 à 0,66, também classificados, respectivamente, como Péssimo e Bom.

Para as séries de temperatura do ar do GHCN e da UDEL foram observados três padrões similares de dispersão: i) tendência de superestimava (Cordeiro) (Figura 4a e 4b), ii) subestimativa (Campos dos Goytacazes, Carmo, Itaperuna e Rio de Janeiro) (Figuras $4 c$ e 4d) ou iii) superestimava/subestimativa, dependendo do valor de temperatura do ar (Angra dos Reis e Resende) (Figura 4e e 4f). Apenas na estação de Santa Maria Madalena os padrões entre os produtos foram diferentes, sendo de 
superestimava para o GHCN e subestimativa para UDEL. Confirma-se também visualmente os resultados dos índices estatísticos ( $r^{2}$ e d de Willmott), que indicaram maior precisão e exatidão das séries de UDEL (Figura 4b, 4d e 4f) em relação ao GHCN (Figura 4a, 4c e 4e) para a maior parte das estações.
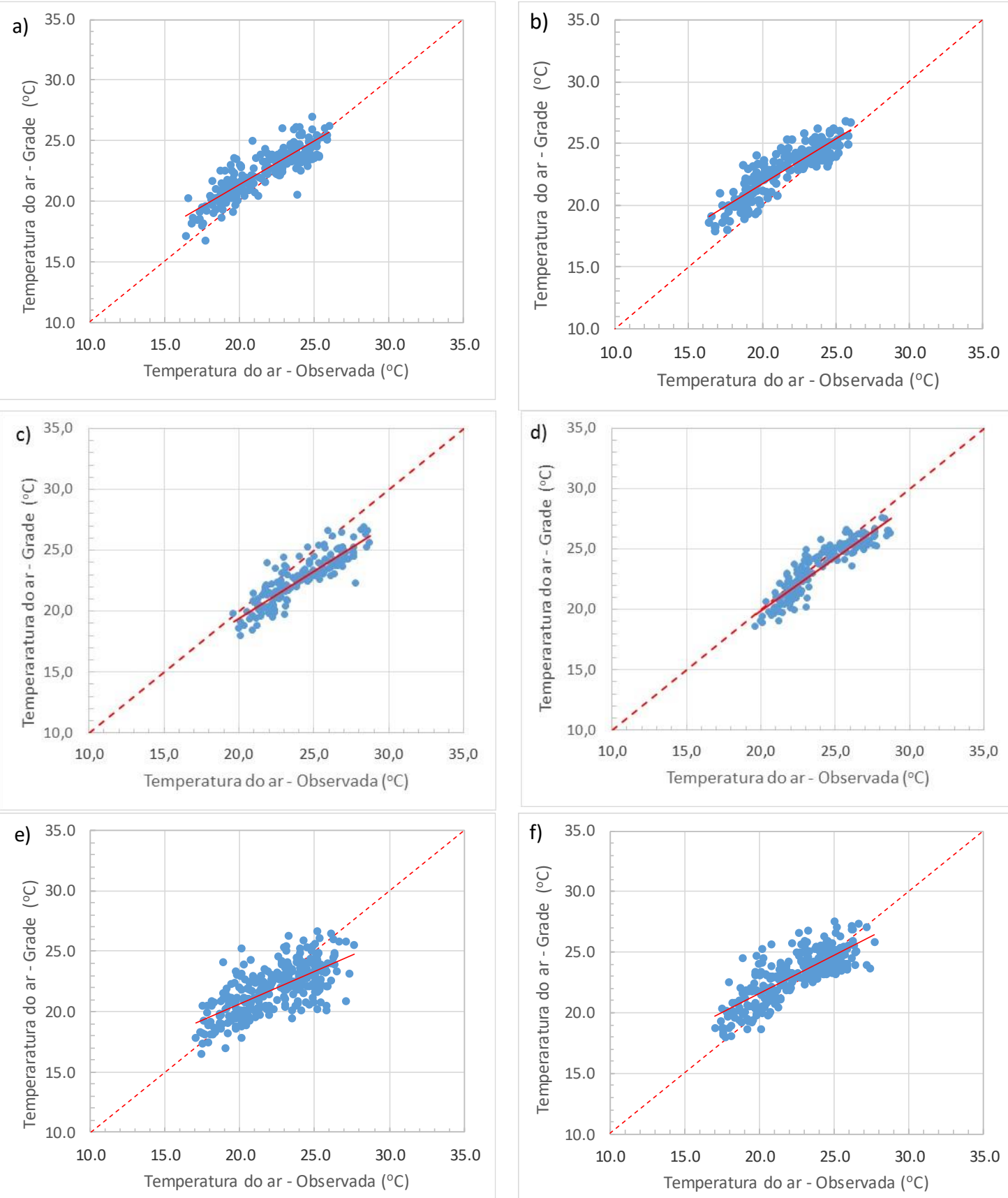

Figura 4 - Relação entre as séries observadas e em grade de temperatura do ar para as estações Cordeiro ( $a, b)$, Itaperuna (c, d) e Resende (e, f) dos produtos em grade do Global Historical Climate Network (a, c, e) e da Universidade de Delaware (b, d, f). 
Para a estação de Cordeiro (padrão i), o erro dos produtos em grade, aumentaram com a diminuição das temperaturas do ar, ou seja, são maiores no inverno. No caso do padrão ii (Campos dos Goytacazes, Carmo, Itaperuna e Rio de Janeiro), os erros tenderam a aumentar com o aumento da temperatura do ar, principalmente, no produto do $\mathrm{GHCN}$, e dessa forma, o verão apresentou menor exatidão dos produtos em grade. Com exceção de Angra, as estações do padrão ii, tem as menores altitude (entre 11 e $280 \mathrm{~m}$ ) entre as estações avaliadas de temperatura do ar. Nas estações do padrão iii) observaram-se os maiores erros no inverno e verão.

No caso da temperatura do ar, a relação entre o RQME, distância e diferença relativa entre a altitude $(\square Z)$ da estação e a do ponto de grade dos produtos do GHCN e UDEL, foi significativa ( $p<0.05$ ), ou seja, essas variáveis não explicaram estatisticamente a variabilidade do erro.

\section{CONCLUSÕES}

Com base nos índices estatísticos, os dados em grade de precipitação para ambos os produtos avaliados (Global Precipitation Climatology Centre e Universidade de Delaware) não apresentam, de forma geral, precisão satisfatória, sendo sua exatidão superior à precisão. O melhor desempenho é das séries do Global Precipitation Climatology em relação à da Universidade de Delaware. Os erros dos produtos em grade aumentam no verão.

Os dados em grade de temperatura do ar da Universidade de Delaware e do Global Historical Climate Network apresentam precisão e exatidão para o estado do Rio de Janeiro, quando comparados com os dados observados, sobressaindo-se o desempenho das séries em grade da Universidade de Delaware. Dependendo da estação e da sua altitude, os maiores erros dos produtos em grade ocorrem no inverno (Cordeiro), no verão (Campos dos Goytacazes, Carmo, Itaperuna e Rio de Janeiro) ou inverno e verão (Angra dos Reis e Resende).

A proximidade do ambiente costeiro resulta em menor precisão e exatidão das séries em grade (Rio de Janeiro e Angra dos Reis) de precipitação e temperatura do ar, independente do produto (GPCC, GHCN ou UDEL), resultado da ausência de estações no oceano, o que compromete a interpolação nas regiões próximas ao ambiente costeiro.

No caso da precipitação, a distância entre o ponto de grade e a estação meteorológica, assim, como as diferenças altitudinais entre esses locais, são fontes de erros.

Os erros associados às séries em grade, principalmente precipitação, restringem estudos do clima regional para o Rio de Janeiro, sendo necessário obter dados em grade com maior resolução espacial e que melhor represente a distribuição espaço-temporal das precipitações e da temperatura do ar no Estado. Sugerem-se para isso estudos baseados em técnicas de refinamento de grade (downscaling) ou métodos de interpolação espacial que considerem o relevo. 


\section{AGRADECIMENTOS}

À Fundação Carlos Chagas Filho de Amparo à Pesquisa do Estado do Rio de Janeiro - FAPERJ pelo auxílio financeiro, processo No - 141.663, e fomento a bolsa do primeiro autor, e ao Conselho Nacional de Desenvolvimento Científico e Tecnológico - CNPq pelo fomento a bolsa do segundo auto.

\section{REFERÊNCIAS BIBLIOGRÁFICAS}

ALVES, E. D. L.; VECCHIA, F. A. S. Análise de diferentes métodos de interpolação para a precipitação pluvial no Estado de Goiás. Acta Scientiarum. Human and Social Sciences, v. 33, n. 2, p. 193-197, 2011.

ANDRIUCCI, L. R.; NETO, J. L. S. Valoração ambiental: uma abordagem teórica das relações entre os estudos climatológicos e as análises econômicas. Revista Brasileira de Climatologia, v. 2, p. 103-120, 2006.

BRITO, T. T.; OLIVEIRA-JUNIOR, J. F.; LYRA, G. B.; GOIS, G.; ZERI, M. Multivariate analysis applied to monthly rainfall over Rio de Janeiro state, Brazil. Meteorology and Atmospheric Physics, p. 1-10, 2016.

CAMARGO, A. P.; SENTELHAS, P. C. Avaliação do desempenho de diferentes métodos de estimativa da evapotranspiração potencial no Estado de São Paulo, Brasil. Revista Brasileira de Agrometeorologia, v. 5, n. 1, p. 89-97, 1997.

CEPERJ - Centro Estatual de Estatísticas, Pesquisas e Formação de Servidores Públicos do Rio de Janeiro. O Estado do Rio de Janeiro e seu Ambiente. Disponível em: http://www.ceperj.rj.gov.br/ceep/info_territorios/ambiente.html. Acesso em: 04 de jun. 2016.

CORREIA, T.P.; PRECINOTO, R.S.; LIMA, A. O.; CRUZ, C. C.; LYRA, G. B.; SANTOS, E. O. Controle de qualidade de séries mensais de precipitação pluvial no estado do Rio de Janeiro. In: XVIII Congresso Brasileiro e VII Reunião Latino-Americana de Agrometeorologia, 2013, Belém. Cenários de mudanças climáticas e a sustentabilidade socioambiental e do agronegócio na Amazônia. Campinas: Sociedade Brasileira de Agrometeorologia, 2013.

DI PIAZZA, A.; LO CONTI, F.; NOTO, L. V.; VIOLA, F.; LA LOGGIA, G. Comparative analysis of different techniques for spatial interpolation of rainfall data to create a serially complete monthly time series of precipitation for Sicily, Italy. International Journal of Applied Earth Observation and Geoinformation, v. 13 , n. 3, p. 396-408, 2011.

JONES, P. D.; MOBERG, A. Hemispheric and Large-Scale Surface Air Temperature Variations: An Extensive Revision and an Update to 2001. Journal of Climate, v. 16, n. 2, 206-223, 2003.

KALNAY, E. et al. The NCEP/NCAR 40-year reanalysis project. Bulletin of American Meteorological Society, v. 77, p. 437-470, 1996.

KANAMITSU, M.; EBISUZAKI, W.; WOOLLEN, J.; YANG, S. K.; HNILO, J. J.; FIORINO, M.; POTTER, G. L. Ncep-doe amip-ii reanalysis ( $r-2)$. Bulletin of the American Meteorological Society, v. 83, n. 11, p. 1631-1643, 2002.

KAWANISHI, T.; TAKAMATSU, H.; KOZU, T.; OKAMOTO, K. I.; KUMAGAI, H. TRMM precipitation radar. In: Geoscience and Remote Sensing Symposium, 
1993. IGARSS'93. Better Understanding of Earth Environment, International. IEEE, 1993. p. 423-425.

KAWANISHI, T. et al. TRMM precipitation radar. Advances in Space Research, $v$. 25, n. 5, p. 969-972, 2000.

KELLER, V. D. J.; TANGUY, M.; PROSDOCIMI, I.; TERRY, J. A.; HITT, O.; COLE, S. J.; FRY, M.; MORRIS, D. G., DIXON, H. CEH-GEAR: $1 \mathrm{~km}$ resolution daily and monthly areal rainfall estimates for the UK for hydrological use. Earth System Science Data Discussions, v. 8, n. 1, p. 143-155, 2015.

KITE, G. W. Frequency and risk analyses in hydrology. Water Resourcers Publications, 1988, $257 \mathrm{p}$.

LAWRIMORE, J. H.; MENNE, M. J.; GLEASON, B. E.; WILLIAMS, C. N.; WUERTZ, D. B.; VOSE, R.S.; RENNIE, J. An overview of the Global Historical Climatology Network monthly mean temperature data set, version 3. Journal Geophysical. Research, v. 116, n. D19, p. 1-18, 2011.

LI, J.; HEAP, A.D. A Review of Spatial Interpolation Methods for Environmental Scientists. Record 2008/023. , Canberra: Geoscience Australia, 2008.

LY, S.; CHARLES, C.; DEGRÉ A. Different methods for spatial interpolation of rainfall data for operational hydrology and hydrological modeling at water shed scale: a review. Biotechnologie, Agronomie, Société et Environnement, v. 17, n. 2, p. 392-406, 2013.

LYRA, G. B.; IBET-LOZADA, B. G.; PIEDADE, S. M. De STEFANO; SEDIYAMA, G. C.; SENTELHAS, P. C.. Regiões homogêneas e funções de distribuição de probabilidade da precipitação pluvial no Estado de Táchira, Venezuela. Pesquisa Agropecuária Brasileira, v. 41, n.2, p. 202-215, 2006.

LYRA, G. B.; OLIVEIRA-JÚNIOR, J. F.; ZERI, M. Cluster analysis applied to the spatial and temporal variability of monthly rainfall in Alagoas state, Northeast of Brazil. International Journal of Climatology, v. 34, n. 13, p. 3546-3558, 2014.

NINYEROLA, M.; PONS, X.; ROURE, J. M. Monthly precipitation mapping of the Iberian Peninsula using spatial interpolation tools implemented in a Geographic Information System. Theoretical and Applied Climatology, v. 89, n. 3, p. 195209, 2006.

MATSUURA, K.; WILLMOTT, C. J. Terrestrial precipitation gridded monthly time series (Version 2.01). Newark: Center for Climatic Research, Department of Geography, University of Delaware, 2009. Disponível em: http://climate.geog.udel.edu/ climate/. Acesso em: 21 de nov. 2014

NOAA/OAR/ESRL PSD - National Centers for Environmental Information Disponível em: http://www.esrl.noaa.gov/psd/. Acesso dia 27 de nov. 2016.

OLIVEIRA-JÚNIOR, J. F. DE; LYRA, G. B.; GÓIS, G.; BRITO, T. T.; MOURA, N. S. H. DE. Análise de Homogeneidade de Séries Pluviométricas para Determinação do Índice de Seca IPP no Estado de Alagoas. Floresta e Ambiente, v. 19, p. 101$112,2012$.

PETERSON, T. C.; VOSE, R. S. An Overview of the Global Historical Climatology Network Temperature Data Base. Bulletin of the American Meteorological Society, v. 78, n. 12, p. 2837-2849, 1997. 
PRECINOTO, R. S.; LEMOS FILHO, G. R.; CORREIA, T.P.; SANTOS, E. O.; LYRA, G. B.; CRUZ, S. M. S. Uso de sistema de pré-processadores para obtenção de séries pluviométricas de qualidade. In: XVIII Congresso Brasileiro e VII Reunião Latino-Americana de Agrometeorologia, 2013, Belém. Cenários de mudanças climáticas e a sustentabilidade socioambiental e do agronegócio na Amazônia. Campinas: Sociedade Brasileira de Agrometeorologia, 2013.

PRECINOTO, R. S.; CORREIA, T.P.; SANTOS, E. O.; LYRA, G. B. Aplicação de regressão linear múltipla para preenchimento de falhas de dados pluviométricos no estado do Rio de Janeiro. In: XVII Congresso Brasileiro de Meteorologia, 2012, Gramado. Incertezas e desafios para a sustentabilidade planetária: o papel da ciência meteorológica. Rio de Janeiro: Sociedade Brasileira de Meteorologia - SBMET, 2012.

SANTOS, A. A. R.; LYRA, G.B.; LIMA, E. P.; SOUZA, J. L.; DELGADO, R.C. Evapotranspiração de referência em função dos extremos da temperatura do ar no estado do Rio de Janeiro. Irriga, v. 21, n. 3, p. 449, 2016.

SCHNEIDER, U. et al. GPCC's new land surface precipitation climatology based on quality-controlled in situ data and its role in quantifying the global water cycle. Theoretical and Applied Climatologyv, 115, n. 1-2, p. 15-40, 2014.

TOSTES, J. O.; LYRA, G. B.; OLIVEIRA- JÚNIOR, J. F.; FRANCELINO, M. R. Assessment of gridded precipitation and air temperature products for the State of Acre, southwestern Amazonia, Brazil. Environmental Earth Sciences, v. 76, n. 4, p. 153, 2017.

WARD JR, J. H. Hierarchical grouping to optimize an objective function. Journal of the American statistical association, v. 58, n. 301, p. 236-244, 1963.

WILLMOTT, C. J. On the validation of models. Physical geography, v. 2, n. 2, p. 184-194, 1981.

WILLMOTT, C. J.; MATSUURA, K.; LEGATES, D. R. Terrestrial air temperature and precipitation: monthly and annual time series (1950-1999). Center for climate research version, v. 1, 2001.

WANG, Y.Q. MeteoInfo: GIS software for meteorological data visualization and analysis. Meteorological Applications, v. 21, n. 2, p. 360-368, 2014.

ZHU, X. ZHANG, M.; WANG, S.; QIANG, F.; ZENG, T.; REN, Z.; DONG, L. Comparison of monthly precipitation derived from high-resolution gridded datasets in arid Xinjiang, central Asia. Quaternary International, v. 358, p. 160$170,2015$. 